\title{
Diminazene Is a Slow Pore Blocker of Acid-Sensing lon Channel 1a $(\mathrm{ASIC1a})^{\mathrm{g}}$
}

\author{
Axel Schmidt, Giulia Rossetti, Sylvia Joussen, and Stefan Gründer \\ Institute of Physiology (A.S., S.J., S.G.), and Department of Oncology, Hematology and Stem Cell Transplantation (G.R.), RWTH \\ Aachen University, Aachen, Germany; and Computational Biomedicine - Institute for Advanced Simulation/Institute of \\ Neuroscience and Medicine, and Jülich Supercomputing Centre, Jülich, Germany (G.R.)
}

Received July 26, 2017; accepted October 10, 2017

\section{ABSTRACT}

Acid-sensing ion channels (ASICs) are neuronal receptors for extracellular protons. They contribute to the excitatory postsynaptic current and to the detection of painful acidosis. Moreover, they are activated during peripheral inflammation and acidosis associated with various neuronal disorders, such as stroke and neuroinflammation, rendering them interesting drug targets. Diminazene aceturate is a small-molecule inhibitor of ASICs with a reported apparent affinity in the low micromolar range, making it an interesting lead compound. It was reported that diminazene accelerates desensitization of ASICs, which was, however, not explained mechanistically. Furthermore, a binding site in a groove of the extracellular domain was proposed but not experimentally verified. In this study, we revisited the mechanism of inhibition by diminazene and its binding site on ASIC1a, the ASIC subunit with the greatest importance in the central nervous system. We show that diminazene slowly blocks ASIC1a, leading to the apparent acceleration of desensitization and underestimating its potency; we show that diminazene indeed has a submicromolar potency at ASIC1a $\left(\mathrm{IC}_{50} 0.3 \mu \mathrm{M}\right)$. Moreover, we show that the inhibition is voltage-dependent and competes with that by amiloride, a pore blocker of ASICs. Finally, we identify by molecular docking a binding site in the ion pore that we confirm by site-directed mutagenesis. In summary, our results show that diminazene blocks ASIC1a by a slow open-channel block and suggest that diminazene is an interesting lead compound for high-affinity blockers of ASICs.

\section{Introduction}

Acid-sensing ion channels (ASICs) are ligand-gated $\mathrm{Na}^{+}$ channels that are opened by extracellular protons (Waldmann et al., 1997; Gründer and Pusch, 2015). They are members of the DEG/ENaC gene family and related to the epithelial $\mathrm{Na}^{+}$channel (ENaC) (Kellenberger and Schild, 2015). In rodents and humans, four ASIC genes encode six major subunits, ASIC1a, ASIC1b, ASIC2a, ASIC2b, ASIC3, and ASIC4, which form different homo- and heteromeric channels. Crystallization of chicken ASIC1 and singlemolecule imaging revealed that ASICs assemble as trimers (Jasti et al., 2007; Bartoi et al., 2014). They have a topology with two transmembrane domains (TMDs), intracellular termini and a large extracellular domain (ECD) (Saugstad et al., 2004), that folds in a complicated three-dimensional structure, which has been compared with a clenched hand

This work was supported by the medical faculty of RWTH Aachen University.

This work was presented in part in abstract form at the following meeting. 96th Annual Meeting of the German Physiological Society; 2017 Mar 16-18; Greifswald, Germany. Vol 219 (Suppl S711), German Physiological Society, Lübeck, Germany.

https://doi.org/10.1124/mol.117.110064.

S This article has supplemental material available at molpharm. aspetjournals.org.
(Jasti et al., 2007). The pore is mainly lined by TMD2 (Li et al., 2011; Baconguis et al., 2014).

ASICs are present in virtually every neuron. In the central nervous system (CNS), the main ASICs are homomeric ASIC1a and heteromeric ASIC1a/2a (Baron et al., 2002; Askwith et al., 2004; Vukicevic and Kellenberger, 2004; Wu et al., 2004) and ASIC1a/2b (Sherwood et al., 2011). ASIC1a is essential for the formation of highly proton-sensitive ASICs in the CNS. In the peripheral nervous system (PNS), all ASIC subunits, except ASIC4, are expressed (Gründer et al., 2000). In the CNS, protons act as a neurotransmitter in synaptic transmission, and activation of ASICs makes a small contribution to the excitatory postsynaptic current (Du et al., 2014; Kreple et al., 2014; González-Inchauspe et al., 2017). In the PNS, activation of ASICs contributes to the detection of metabolic and painful acidosis (Molliver et al., 2005; Deval et al., 2008; Diochot et al., 2012; Deval and Lingueglia, 2015).

It is also increasingly recognized that ASICs make important contributions to different pathophysiological states (Wemmie et al., 2013; Huang et al., 2015). For example, ASICs are activated during ischemia associated with stroke, increasing neurodegeneration (Xiong et al., 2004; McCarthy et al., 2015; Chassagnon et al., 2017). Moreover, ASICs contribute to axonal degeneration in an animal model of neuroinflammation (Friese et al., 2007). In the PNS, ASICs contribute to inflammatory and neuropathic pain

ABBREVIATIONS: A-317567, (C-\{6-[2- (1-Isopropyl-2-methyl-1,2,3,4-tetrahydro-isoquinolin-7-yl)-cyclopropyl]-naphthalen-2-yl\}-methanediamine); ASIC, acid-sensing ion channel; BASIC, bile acid-sensitive ion channel; CNS, central nervous system; ECD, extracellular domain; $\mathrm{ENaC}$, epithelial $\mathrm{Na}^{+}$channel; $\mathrm{HyNaC}$, peptide-gated ion channels from Hydra; MES, 4-morpholineethanesulfonic acid; PLIF, protein ligand interaction fingerprint; PNS, peripheral nervous system; rASIC1a, rat ASIC1a; TMD, transmembrane domain; wt, wild type. 
(Mazzuca et al., 2007; Deval et al., 2008; Yen et al., 2009; Deval and Lingueglia, 2015; Diochot et al., 2016). All these conditions make ASICs interesting drug targets. Thus, inhibitors of ASICs are not only useful tools for understanding the physiologic roles of ASICs but also of clinical relevance for the treatment of different neuronal disorders (Baron and Lingueglia, 2015; Rash, 2017).

The prototypical blocker of ASICs is the diuretic amiloride, characterized by a guanidine moiety. Amiloride inhibits all ASICs, but is not specific to them, also inhibiting, among other molecules, the ENaC and the $\mathrm{Na}^{+} / \mathrm{H}^{+}$exchanger (Kleyman and Cragoe, 1988). Moreover, potency of amiloride at ASICs is 100-fold lower $\left(\mathrm{IC}_{50} \sim 10 \mu \mathrm{M}\right.$ ) (Waldmann et al., 1997) than at $\mathrm{ENaC}\left(\mathrm{IC}_{50} 0.1 \mu \mathrm{M}\right)$ (Canessa et al., 1994). For ENaC, amiloride is an open-channel blocker with a binding site within the pore (Schild et al., 1997). Cocrystals of chicken ASIC1 with amiloride confirmed a binding site in the ion pore for ASIC1 (Baconguis et al., 2014). A small-molecule inhibitor of ASICs lacking the guanidine moiety is A-317567 (C-\{6-[2(1-Isopropyl-2-methyl-1,2,3,4-tetrahydro-isoquinolin-7-yl)cyclopropyl]-naphthalen-2-yl\}-methanediamine) (Dubé et al., 2005) with a potency at ASICs that is comparable to that of amiloride $\left(\mathrm{IC}_{50} 2-30 \mu \mathrm{M}\right)$. Nafamostat mesilate, a protease inhibitor, is another small-molecule inhibitor with a potency of $15 \mu \mathrm{M}$ at ASIC1a and $2 \mu \mathrm{M}$ at ASIC3 (Ugawa et al., 2007).

One of the most potent small-molecule inhibitors of ASICs is diminazene, a diarylamidine that is used as an antiprotozoal drug in veterinary medicine (Chen et al., 2010a). It inhibits all ASICs, but not ENaC; apparent affinity for ASIC1a was reported to be $3 \mu \mathrm{M}$ for ASIC1a, but was higher for ASIC1b and ASIC3 (Chen et al., 2010b). In addition, it was reported that diminazene accelerated ASIC desensitization, and molecular docking predicted a binding site in a groove of the ECD (Chen et al., 2010b). Due to the high potency of diminazene at ASICs, it is an interesting lead compound for the rational design of new ASIC inhibitors. Such an approach would greatly benefit from the molecular identification of the binding site of diminazene on ASICs (Chen et al., 2010a). In this study, we analyzed in detail the mechanism of block by diminazene and its binding site on ASIC1a. We found that diminazene slowly binds to a binding site within the ion pore of open ASIC1a.

\section{Materials and Methods}

Chemicals. All chemicals used were purchased from Sigma-Aldrich (St. Louis, MO), VWR (Radnor, PA), or Carl Roth (Karlsruhe, Germany).

Site-Directed Mutagenesis and cRNA Synthesis. The plasmid containing rat ASIC1a (rASIC1a) cDNA used in this study was described previously (Bässler et al., 2001). Mutants of rASIC1a were generated by site-directed mutagenesis according to the QuikChange protocol (Agilent, Santa Clara, CA). All mutations were verified by sequencing of the entire cDNA. For cRNA synthesis, plasmids were linearized. Capped cRNA was synthesized in vitro with the mMessage mMachine kit using the SP6 RNA Polymerase (Ambion, Austin, TX).

Xenopus laevis Oocyte Preparation, Injection, and Incubation. Animal care and experiments were conducted according to protocols approved by the State Office for Nature, Environment, and Consumer Protection of the state North Rhine-Westphalia in Germany. Ovaries were surgically removed from female Xenopus laevis frogs as previously described (Joeres et al., 2016), and oocytes were separated by enzymatic treatment with collagenase type 2 (Worthington Biochemical Corporation, Lakewood, NJ) for 2 hours. Stage V or VI oocytes were injected with 0.03-0.08 ng of cRNA coding for wild-type rASIC1a or with the following amounts of cRNA coding for ASIC1a mutants: 0.08-16 ng G431C, 16 ng G435C, 0.08-16 ng Q436C, 16 ng L439C, 0.03-0.08 ng F440C, or
0.03-0.08 ng I441C. Oocytes were then incubated for 24-72 hours at $19^{\circ} \mathrm{C}$ in oocyte Ringer 2 medium (in millimolars: $82.5 \mathrm{NaCl}, 2.5 \mathrm{KCl}, 1.0$ $\mathrm{Na}_{2} \mathrm{HPO}_{4}, 5.0 \mathrm{HEPES}, 1.0 \mathrm{MgCl}_{2}, 1.0 \mathrm{CaCl}_{2}, 0.5 \mathrm{~g} / \mathrm{l}$ polyvinylpyrrolidone, $1000 \mathrm{U} / \mathrm{l}$ penicillin, and $10 \mathrm{mg} / \mathrm{l}$ streptomycin; $\mathrm{pH}$ was adjusted to $\mathrm{pH}$ 7.3).

Electrophysiology. Two-electrode voltage clamp of Xenopus oocytes was performed at ambient temperature $\left(21-23^{\circ} \mathrm{C}\right)$ with a Turbo Tec 03X amplifier (npi electronic, Tamm, Germany). Micropipettes filled with $3 \mathrm{M} \mathrm{KCl}$ and a resistance of $0.5-3 \mathrm{MOhm}$ were used as intracellular electrodes. Fast solution exchange was enabled by a pump-driven system (Madeja et al., 1995) controlled by the software Cellworks (version 5.5.1; npi electronic). This solution-exchange system is optimized to achieve rapid solution exchange but does not provide constant superfusion of the oocytes. At high concentrations of an inhibitor, traces of the inhibitor might, therefore, still be present in the bath after washout. Residual diminazene likely explains the kink in some of the traces in Fig. 2. However, since control traces were similarly affected, this does not influence the results of the experiment. The holding potential was $-70 \mathrm{mV}$, and currents were digitized at $200 \mathrm{~Hz}$, if not stated otherwise. Bath solutions contained the following (in millimolars): $140 \mathrm{NaCl}, 10 \mathrm{HEPES}, 1.8 \mathrm{CaCl}_{2}$, and $1 \mathrm{MgCl}_{2}$ (pH was adjusted with $\mathrm{NaOH}$ ); 4-morpholineethanesulfonic acid (MES) replaced HEPES for $\mathrm{pH}<6.6$. Diminazene aceturate or amiloride hydrochloride was added to the bath solution as indicated. A solution with conditioning $\mathrm{pH} 7.4$ was applied for 60 seconds prior to each activation. For all experiments, oocytes from at least two different frogs were used.

Data Analysis. Electrophysiological data were displayed in Cellworks Reader (version 3.7; npi electronic) and quantified in either Cellworks Reader or Igor Pro (version 5.0.3; WaveMetrics, Lake Oswego, OR). Subsequent statistical analysis was performed in Excel (Microsoft, Redmond, WA). Results are reported as the mean \pm S.E.M. Student's paired or unpaired $t$ test, as appropriate, was used to determine $P$ values. When multiple tests were conducted on the same data set, we used the Bonferroni correction, multiplying individual $P$ values by the number of tests. Graphs and electrophysiological traces were plotted with Igor Pro.

Concentration-response curves were fit by a Hill function:

$$
I_{\mathrm{X}}=I_{0} /\left[1+\left([x] / K_{\mathrm{i}}\right)^{H}\right]
$$

where $I_{\mathrm{x}}$ represents the current at a given concentration of inhibitor $x$, $I_{0}$ is the current in the absence of inhibitor, $[x]$ is the concentration of the inhibitor $x, K_{\mathrm{i}}$ is the concentration at which $50 \%$ of the current was inhibited (apparent inhibitory constant), and $H$ is the Hill coefficient. Values for $K_{\mathrm{i}}$ and $H$ indicated in the text are means \pm S.E.M. of individual measurements. Fits depicted in figures were fit to the data points plotted with $I_{0}$ fixed to 1 , for comparability.

Hill coefficients 1 second postpeak were close to 1 (1.23 \pm 0.06 for diminazene and $1.07 \pm 0.04$ for amiloride; see "Results," Fig. 3). To estimate $K_{\mathrm{i}}$ at different holding potentials, we therefore fixed $H$ to 1 and used a simplified Hill equation:

$$
K_{\mathrm{i}}=[x] I_{\mathrm{x}} /\left(I_{0}-I_{\mathrm{x}}\right) .
$$

Apparent inhibitory constants $\left(K_{\mathrm{i}}\right)$ at different holding potentials were subsequently fit by the Woodhull model of channel block (Woodhull, 1973):

$$
K_{\mathrm{i}}(V)=K_{\mathrm{i}}(0 \mathrm{mV}) \exp \left(z^{\prime} F^{\prime} V / R T\right)
$$

where $V$ represents the membrane potential, $K_{\mathrm{i}}(0 \mathrm{mV})$ is the inhibitory constant at $0 \mathrm{mV}, F$ is the Faraday constant, $R$ is the gas constant, $T$ is the absolute temperature, and $z^{\prime}$ is the product of the valence of the inhibitor and the fraction of the membrane potential $(\delta)$ acting on the inhibitor. Thus, for diminazene, $\delta$ can be calculated by $\delta=z^{\prime} / 2$, and by $\delta=z^{\prime}$ for amiloride.

Time constants of current decay $(\tau)$ were determined by fitting current traces with an exponential function using Igor Pro:

$$
I(\mathrm{t})=I_{0}+A e^{(-t / \tau)}
$$

where $I(\mathrm{t})$ represents the current at time $\mathrm{t}, I_{0}$ is the basal current, and $A$ is the amplitude of the desensitizing current component. 
Homology Modeling and Molecular Docking. Rat ASIC1a homology models were built with MODELLER 9.16 (Andrej Sali; https://salilab.org/modeller/) (Sali and Blundell, 1993) based on the crystal structure of the chicken ASIC1 open conformation (Protein Data Bank: 4NTW) (Baconguis et al., 2014). The two sequences have $89.6 \%$ of identity and $94.6 \%$ of similarity along the overall structure, and they are identical in the considered binding site according to Needle Pairwise Sequence Alignment of the Emboss Web server (http://www.ebi.ac.uk/Tools/psa/ emboss_needle/). The best out of 500 models was selected based on the discrete optimized protein energy scoring function (Shen and Sali, 2006) with a stereochemical quality of $98 \%$ of residues in the favored region of the Ramachandran plot (Lovell et al., 2003).

Diminazene and amiloride were structurally preprocessed using LigPrep from the Schrödinger Suite (Schrödinger, Cambridge, MA) (Sastry et al., 2013). Molecular docking was conducted on the best ASIC1a model preprocessed with the Protein Preparation Wizard from the Schrödinger Suite (Jacobson et al., 2002; Shelley et al., 2007; Greenwood et al., 2010; Sastry et al., 2013). The protonation states of each side chain were generated using the software Epik (Schrödinger) for $\mathrm{pH}=7$ (Shelley et al., 2007; Greenwood et al., 2010). Protein energy minimization was performed using the OPLS3 Force Field (Schrödinger) (Jorgensen et al., 1996; Harder et al., 2016).

Glide 75013 (Schrödinger) (Friesner et al., 2004; Halgren et al., 2004) was used for all docking calculations. Internal and external receptor grid boxes of $20 \times 20 \times 20$ and $40 \times 40 \times 40 \AA$ were centered in the transmembrane region. Initially, a standard precision Glide docking followed by extra precision refinement was carried out, generating at least two poses per docked molecule. Ligands are often known to induce conformational changes in the active site upon binding. We therefore used the Schrödinger induced fit docking protocol (Sherman et al., 2006) to account for these changes and to refine our initial docking. The receptor grid center was specified from the bound ligand of the first docking. In the first stage of the induced fit docking protocol, softened-potential docking is performed to generate 20 initial poses. The scaling factors to soften the potentials of the receptors and ligands were set to 0.5 in both cases. A $2.5-\mathrm{kcal} / \mathrm{mol}$ energy window was used for ligand conformational sampling. For each of the top 20 poses (with respect to GlideScore) from the initial softened-potential docking step, all residues within $5.0 \AA$ of ligand poses were refined using the Prime molecular dynamics.

Refinement was performed with the Prime package (Schrödinger) to accommodate the ligand by reorienting nearby side chains. Prime uses the OPLS parameter set (Jorgensen et al., 1996; Harder et al., 2016) and a surface generalized born implicit solvent model (Ghosh et al., 1998; Gallicchio et al., 2002). The complexes were ranked by Prime energy (molecular mechanics plus solvation), and those within $30 \mathrm{kcal} / \mathrm{mol}$ of the minimum energy structure were passed through for a final round of Glide docking and scoring. This $30-\mathrm{kcal} / \mathrm{mol}$ window was set prior to running; it appears that a $20-\mathrm{kcal} / \mathrm{mol}$ window would have been sufficiently large to capture all good poses from the initial docking step. The ligands were then redocked into their corresponding receptor structures using extra precision scoring in Glide. Each docking result was analyzed by comparing the Glide Docking and eModel scores. The first may be used for comparing different ligands, but the second score is suitable to rank different conformations of the same ligand.

\section{Results}

Diminazene Reversibly Blocks rASIC1a in Its Open State. To investigate the molecular mechanism of ASIC1a inhibition by diminazene, we studied its effect on rat ASIC1a expressed in Xenopus oocytes. First, we activated ASIC1a repeatedly by $\mathrm{pH}$ decreases from $\mathrm{pH} 7.4$ to $\mathrm{pH} 6.5$ with $3 \mu \mathrm{M}$ diminazene present at various times (Fig. 1). When diminazene was preapplied at $\mathrm{pH} 7.4$ for 60 seconds and coapplied with $\mathrm{pH} 6.5$, peak current amplitudes were reduced to $29 \% \pm 3 \%$ (Fig. 1 , activation ii) relative to the control activation (Fig. 1, activation i; ii vs. i, $P=0.002$ ). As previously described (Chen et al., 2010b), the presence of diminazene also led to an acceleration of current decline such that almost the whole current was inhibited 1 second after activation (Fig. 1B). When diminazene was only coapplied with $\mathrm{pH}$ 6.5, peak current amplitudes were reduced to $32 \% \pm 4 \%$ of control (Fig. 1, activation iv; iv vs. i, $P<0.001 ; n=10$ ) and currents still declined within the first second after low-pH activation. This reduction was not significantly different from the reduction when diminazene was also preapplied to closed channels (ii vs. iv, $P=1$ ), showing that binding of diminazene to the open state is sufficient for inhibition, which would be expected, for example, when diminazene either has no access to its binding site on ASIC1a in the closed state or when diminazene binding in the closed state does not inhibit the channel.

When we applied diminazene to ASIC1a in the open conformation, 3 seconds after activation by $\mathrm{pH} 6.5$, it rapidly (within $\sim 1$ second) inhibited the channel (Fig. 1, activation iii) such that current amplitudes measured 7 seconds after the
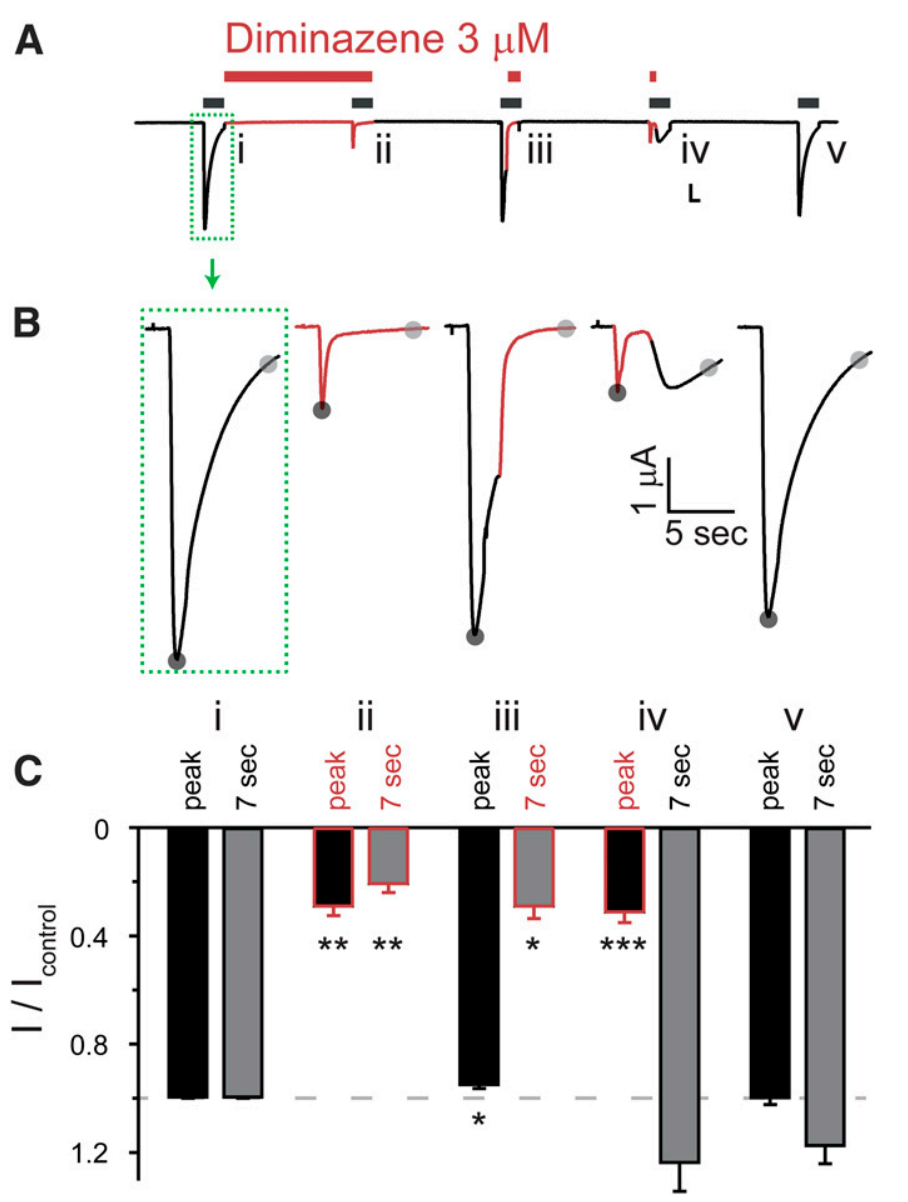

Fig. 1. Diminazene reversibly blocks rASIC1a in its open state. (A) Representative current trace of an rASIC1a-expressing oocyte activated by $\mathrm{pH} 6.5$ for 10 seconds (black bars); conditioning $\mathrm{pH} 7.4$ was applied for 60 seconds. Diminazene $(3 \mu \mathrm{M})$ was added to the bath solution during the following time intervals (indicated by red current traces and red bars): peak ii: during 60 seconds of $\mathrm{pH} 7.4$ and 10 seconds of $\mathrm{pH} 6.5$; peak iii: during the last 7 seconds of $\mathrm{pH} 6.5$; peak iv: during the first 3 seconds of $\mathrm{pH}$ 6.5. (B) Magnification of ASIC currents from (A). (C) Quantification of peak currents [peak; dark-gray dots in (B)] and currents 7 seconds postpeak [7 sec; light-gray dots in (B)] of activations ii - $\mathrm{v}$ relative to the first activation with pH 6.5 (i). $* P<0.05 ; * * P<0.01$; ***P $<0.001$ (paired Student's $t$ test followed by Bonferroni correction for multiple comparisons). Error bars represent S.E.M.; $n=9-10$. 
activation by $\mathrm{pH} 6.5$ were reduced to $29 \% \pm 4 \%$ compared with control (iii vs. i, $P=0.03 ; n=9$ ). Continuous pre- and coapplication of diminazene did not reduce currents 7 seconds postpeak more strongly (Fig. 1 ii; $21 \% \pm 3 \%$; ii vs. i, $P=0.01$; ii vs. iii, $P=0.3 ; n=10$ ), further suggesting that diminazene mainly acts on the open conformation of ASIC1a.

The accelerated current decline in the presence of diminazene (for example, see activation ii in Fig. 1B) was interpreted as a facilitated desensitization (Chen et al., 2010b). When we activated rASIC1a by $\mathrm{pH} 6.5$ in the initial presence of diminazene and 3 seconds later applied $\mathrm{pH} 6.5$ without diminazene (Fig. 1; activation iv), the removal of diminazene induced, however, a rebound of current (current 7 seconds postpeak, $124 \% \pm 10 \%$ of activation i; iv vs. i, $P=0.81 ; n=10$ ), excluding that diminazene shifted ASIC1a in the desensitized state. It rather suggests that a fraction of channels were still in the open state but inhibited by diminazene, and that removal of diminazene removed inhibition. This interpretation suggests that diminazene acts as a slow open-channel blocker, but we cannot completely rule out complex gating modifications by diminazene. Taken together, our results so far suggest that diminazene inhibits ASIC1a in its open state, probably through an open-channel block with slow kinetics, explaining the apparently faster desensitization.

Diminazene Delays Desensitization of ASIC1a. If diminazene indeed blocked ASIC1a by binding to its open conformation, it might trap ASIC1a in the open conformation, slowing down desensitization (rather than facilitating it). To test if this was the case, we activated ASIC1a by $\mathrm{pH} 6.5$ in the presence of $30 \mu \mathrm{M}$ diminazene and quantified currents after 12.5 seconds, when usually a large fraction of channels is desensitized (Fig. 2A). When we quantified control currents without diminazene after 12.5 seconds of $\mathrm{pH} 6.5$ application, they were reduced to $7.8 \% \pm 1.2 \%$ of the peak current (Fig. 2A, black trace; $n=13$ ). This value was indeed significantly increased by the presence of diminazene. Three seconds of diminazene application at the beginning of the activation with $\mathrm{pH} 6.5$ increased the current after 12.5 seconds to $10.9 \% \pm 1.4 \%$ of the peak amplitude $(P=0.01 ; n=13)$, and 10 seconds of diminazene application further increased it to $17.5 \% \pm 2.8 \%(P=0.02 ; n=13)$. The increase of the current amplitude in the partial presence of diminazene demonstrates that diminazene delays ASIC1a desensitization and is therefore in agreement with the idea that it binds to and stabilizes the open conformation of ASIC1a.

After 27.5 seconds of $\mathrm{pH} 6.5$ application, control currents in the absence of diminazene were almost completely reduced to $0.9 \% \pm 0.2 \%$ of the peak amplitude $(n=13)$. When diminazene was present at the beginning of the $\mathrm{pH} 6.5$ application for 3,10 , or 25 seconds, current amplitudes were only slightly increased $(0.9 \% \pm 0.2 \%, 1.3 \% \pm 0.3 \%$, and $2.6 \% \pm 0.6 \%$, respectively; $P=0.03$ for 25 seconds; $n=11-13)$. Thus, diminazene can delay, but not prevent, desensitization of ASIC1a, as would be expected if diminazene binds to and unbinds from its binding site on ASIC1a, allowing channels to gradually "escape" into the desensitized state also in the presence of diminazene.

Diminazene Has a Submicromolar Potency at ASIC1a. A slow inhibition implies that the full inhibition by diminazene is not immediately reached, and that the reduction of peak current amplitudes underestimates the potency of diminazene. We therefore re-examined the apparent affinity
A

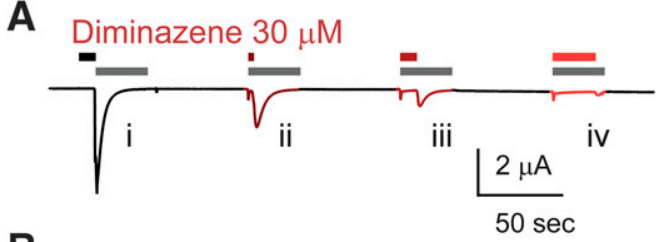

B Diminazene $30 \mu \mathrm{M}$

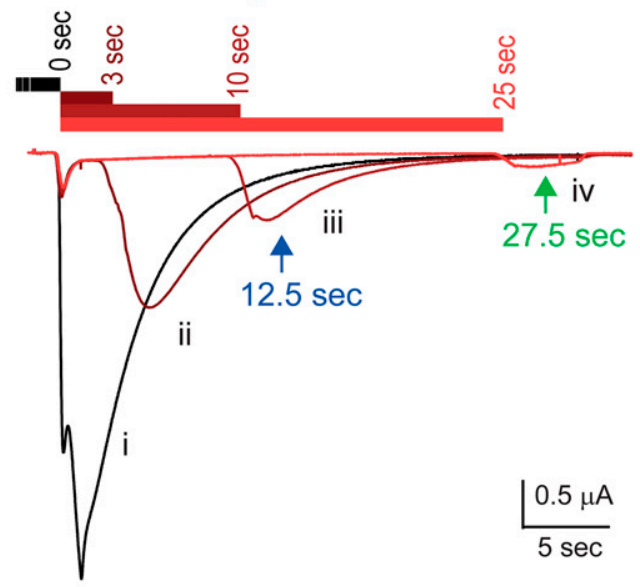

C Duration of inhibition by diminazene (sec)

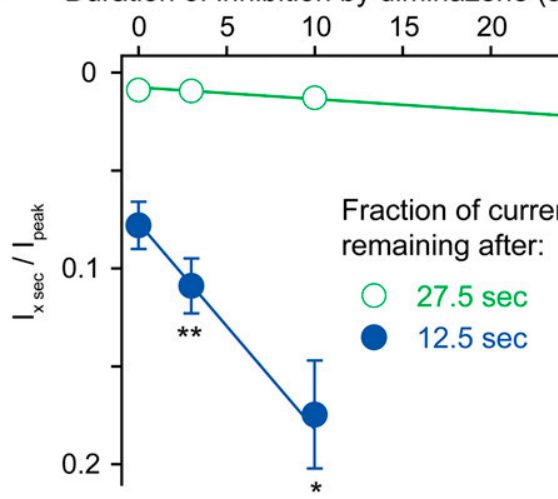

Fig. 2. Diminazene slows ASIC1a desensitization. (A) Representative current trace of an rASIC1a-expressing oocyte activated by pH 6.5 (gray bars) when $30 \mu \mathrm{M}$ diminazene was present for 3,10 , or 25 seconds, respectively, as indicated by the red bars (peaks ii-iv). As control, diminazene was preapplied (black bar; peak i). (B) Magnification of $\mathrm{pH}$ 6.5-activated currents from (A). We attribute the kink in some of the traces to incomplete washout of the inhibitor, which was similar in all measurements, however. (C) Currents remaining 12.5 or 27.5 seconds after the peak normalized to the peak current of the control activation. Data were fit by a linear function; ${ }^{*} P<0.05$; ${ }^{*} P<0.01$ (paired Student's $t$ test followed by Bonferroni correction). Error bars represent S.E.M.; $n=12-14$.

of diminazene for ASIC1a. We pre- and coapplied increasing diminazene concentrations at $\mathrm{pH} 6.5$ to rASIC1a-expressing oocytes (Fig. 3A) and determined concentration-response curves for peak currents, currents 1 second postpeak, and currents 3 seconds postpeak. Currents 1 and 3 seconds postpeak were chosen because $3 \mu \mathrm{M}$ diminazene accelerated the current decay of ASIC1a from $2.26 \pm 0.28$ to $0.55 \pm 0.08$ seconds $(n=9$; fits conducted on activation $\mathrm{i}$ and ii of Fig. 1; one outlier removed). Therefore, current amplitudes at these times represent the diminazene effect more faithfully than peak currents. The apparent affinity for diminazene increased from $0.94 \pm 0.18 \mu \mathrm{M}$ for peak currents to $0.30 \pm 0.07 \mu \mathrm{M}$ for currents 1 second postpeak $(P=0.003 ; n=12)$ and to $0.21 \pm 0.04 \mu \mathrm{M}$ for currents 3 seconds postpeak $(P=0.002$; Fig. $3 \mathrm{C})$. We conclude that the 
A

\section{Diminazene $(\mu \mathrm{M})$}

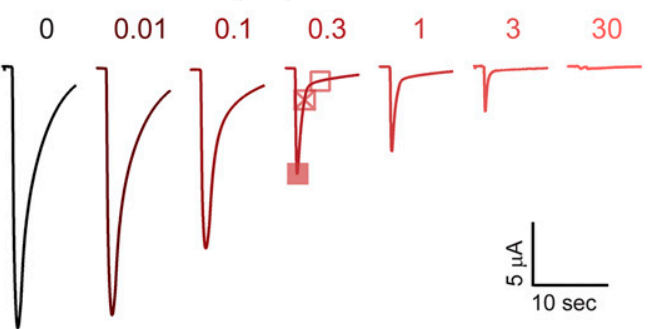

B

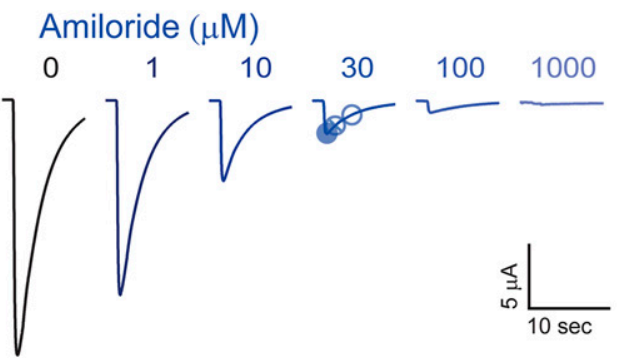

C
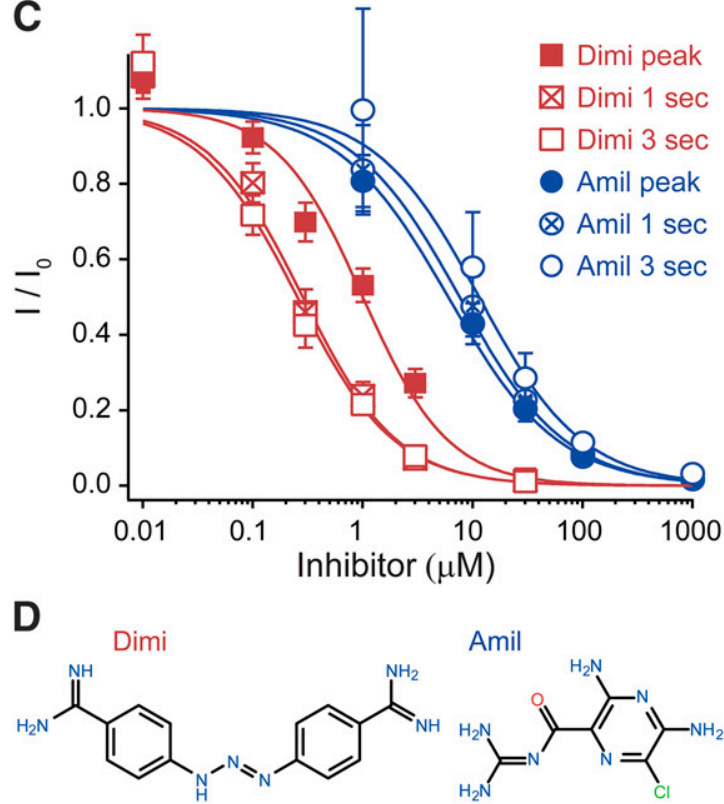

Fig. 3. Diminazene has a submicromolar potency. (A and B) Representative currents elicited by $\mathrm{pH} 6.5$ in an rASIC1a-expressing oocyte. Either diminazene (A) or amiloride (B) was pre- and coapplied at the concentrations indicated. (C) Concentration-response curves of diminazene (Dimi, red) and amiloride (Amil, blue) determined for peak currents and for currents 1 or 3 seconds postpeak [as indicated by the corresponding symbols in (A) and (B)]. Data points displayed were fit to a Hill function. Currents were normalized to the first activation without inhibitor. Error bars represent S.E.M.; $n=12$. (D) Chemical structures of diminazene and amiloride, created with Open Babel 2.3.2 (http://openbabel.org/) (O’Boyle et al., 2011).

use of peak currents for diminazene concentration-response curves indeed underestimates the apparent affinity of diminazene.

In contrast to diminazene, the pore blocker amiloride does not enhance current decay, suggesting an "immediate," fast block. In line with this, potencies of amiloride were indeed similar irrespective of whether they were determined at peak, 1 , or 3 seconds postpeak (Fig. 3B and C; peak: $9.6 \pm 1.3 \mu \mathrm{M}$; 1 second: $11.8 \pm 1.9 \mu \mathrm{M}$; 3 seconds: $14.1 \pm 2.7 \mu \mathrm{M}$; peak vs. 1 second, $P=0.07$; peak vs. 3 seconds, $P=0.09 ; n=12$ ). Thus, concentration-response curves based on peak currents appropriately describe the amiloride potency.

We also determined apparent affinity for diminazene at the more acidic $\mathrm{pH} 5.5$, revealing that diminazene also accelerated the current decline at pH 5.5 (Supplemental Fig. 1A). In agreement, the apparent affinity was higher when determined for currents 1 or 3 seconds postpeak than for peak currents (Supplemental Fig. 1B). Overall, apparent affinity for diminazene was lower at $\mathrm{pH} 5.5$ than at $\mathrm{pH} 6.5$ (peak: $3.49 \pm 1.13 \mu \mathrm{M}, P=0.02$; 1 second: $0.45 \pm 0.05 \mu \mathrm{M}, P=0.11$; 3 seconds: $0.39 \pm 0.03 \mu \mathrm{M}$, $P=0.003, t$ test; $n=9-10$ ), demonstrating some $\mathrm{pH}$ dependency of the diminazene affinity.

Using currents 1 or 3 seconds postpeak for determining the diminazene potency reveals that diminazene is one of the most potent small-molecule inhibitors known for ASIC1a. For all following experiments, we quantified diminazene effects 1 second postpeak.

Amiloride and Diminazene Compete for rASIC1a Inhibition. Slow inhibition of ASIC1a in the open conformation suggests that diminazene might inhibit ASIC1a by a block of the open pore. As amiloride is a pore blocker of DEG/ENaCs (Palmer, 1984; Schild et al., 1997; Baconguis et al., 2014), we next asked whether diminazene competes with amiloride for a common binding site on ASIC1a. To test for a competitive interaction, we activated ASIC1a by $\mathrm{pH} 6.5$ in the presence of $80 \mu \mathrm{M}$ amiloride, which will inhibit $\sim 90 \%$ of the current (Fig. 3C), and pre- and coapplied increasing diminazene concentrations (Fig. 4A). The additional presence of amiloride will increase the total inhibition of the channel, but in the case of competition for a common binding site, it should also decrease the apparent affinity for diminazene. The presence of amiloride indeed shifted the concentration-response curve of diminazene to the right (from $0.30 \pm 0.07$ to $6.0 \pm 0.7 \mu \mathrm{M}$ for currents 1 second postpeak; Fig. 4B). Assuming the competition of two inhibitors for a common binding site, the apparent inhibitory constant $\left(K_{\mathrm{i}}\right)$ of diminazene in the absence $\left(K_{\mathrm{i} \text {,dimi }}\right)$ and in the presence of amiloride $\left(K_{\mathrm{i} \text {,app }}\right)$ can be related by the equation $K_{\mathrm{i} \text {,app }}=K_{\mathrm{i}, \text { dimi }}\left(1+[\mathrm{amil}] / K_{\mathrm{i} \text {,amil }}\right)$. Using $K_{\mathrm{i}, \text { dimi }}=0.3 \mu \mathrm{M}, K_{\mathrm{i} \text {,amil }}=$ $11.8 \mu \mathrm{M}$ (Fig. $3 ; n=13$ ), and [amil] $=80 \mu \mathrm{M}$, a theoretical value $\left(K_{\mathrm{i} \text {,app }}=2.3 \mu \mathrm{M}\right)$ can be calculated that compares well with the experimentally determined value of $6.0 \mu \mathrm{M}$. Thus, these results indicate that both molecules compete for a common binding site in the ASIC1a pore.

Inhibition of ASIC1a by Amiloride and by Diminazene is Voltage-Dependent. To test directly if diminazene binds to the pore of ASIC1a, we tested if the inhibition of ASIC1a by diminazene is voltage-dependent. We activated ASIC1a with $\mathrm{pH} 6.5$ at holding potentials between -100 and $+60 \mathrm{mV}$ in the presence and absence of $3 \mu \mathrm{M}$ diminazene (Fig. 5A). The extent of inhibition of ASIC1a currents indeed depended on the membrane potential, with inhibition being most pronounced at $-100 \mathrm{mV}$ (Fig. 5B). More positive membrane potentials reduced the inhibition by diminazene, and at potentials more positive than $0 \mathrm{mV}$, diminazene almost did not inhibit ASIC1a (Fig. 5A and B). Similar to the magnitude of the inhibition, the accelerated current decline was also most pronounced at negative membrane potentials (Fig. 5A).

Since the Hill coefficient for the diminazene block of ASIC1a was close to 1 for currents 1 second postpeak $(1.23 \pm 0.06, n=12$, data from Fig. 3), we estimated inhibitory constants $\left(K_{\mathrm{i}}\right)$ at each holding potential by a simplified formula derived from the Hill equation assuming a Hill coefficient of $1 . K_{\mathrm{i}}$ values of diminazene 
A

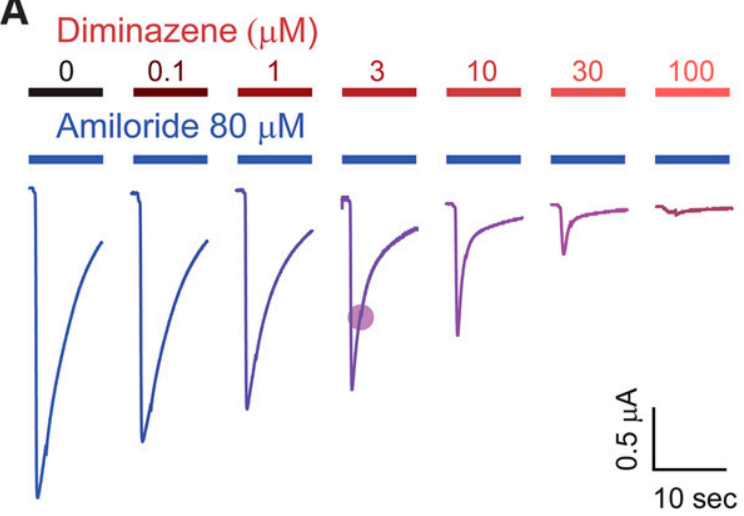

B

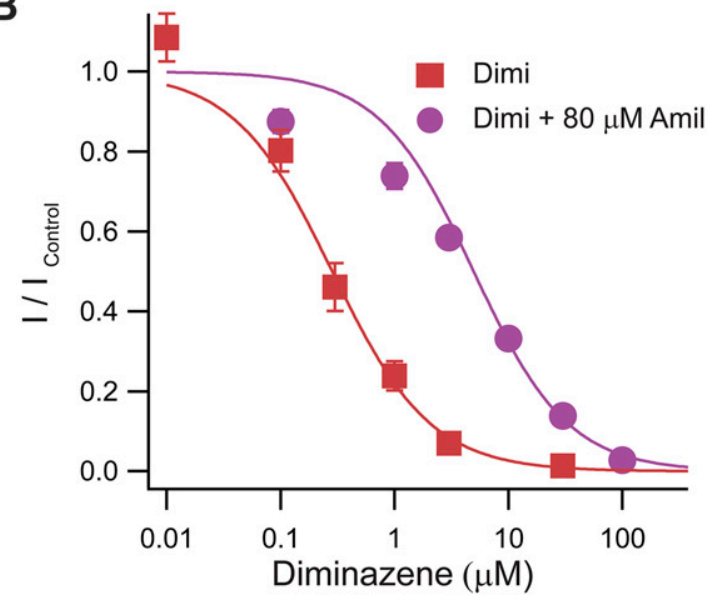

Fig. 4. Amiloride and diminazene compete for rASIC1a inhibition. (A) Representative currents elicited by $\mathrm{pH} 6.5$ in an rASIC1a-expressing oocyte in the presence of $80 \mu \mathrm{M}$ amiloride and increasing concentrations of diminazene. (B) Concentration-response curves for diminazene alone (dimi, red) and for diminazene in the presence of $80 \mu \mathrm{M}$ amiloride (dimi $+80 \mu \mathrm{M}$ amil, purple) for currents 1 second postpeak. Data displayed were fit to a Hill function. Currents were normalized to the first activation without diminazene. Error bars represent S.E.M.

for rASIC1a were clearly voltage-dependent and could be fit by the Woodhull model (see Materials and Methods) for voltagedependent channel block (Fig. 5C). With the Woodhull model, the proportion of the membrane electric field experienced by a molecule can be estimated by the value $\delta$, which was $0.32 \pm 0.03$ for diminazene, determined 1 second postpeak.

To compare the behavior of diminazene and amiloride at different membrane potentials, we repeated the same experiment with $80 \mu \mathrm{M}$ amiloride instead of diminazene. As shown previously by others (Dorofeeva et al., 2008), the inhibition by amiloride was also dependent on the membrane potential and strongest at $-100 \mathrm{mV}$ (Fig. 5D-F). The proportion of the membrane electric field $(\delta)$ sensed by amiloride was $0.51 \pm$ 0.02 (determined 1 second postpeak). Thus, both drugs seem to experience similar proportions of the membrane electric field and should therefore bind at a similar position relative to the membrane electric field. Voltage-dependent block of ASIC1a by diminazene and amiloride is in line with an overlapping binding site of the two inhibitors within the ASIC1a pore.

Molecular Docking of Diminazene and Amiloride to a Homology Model of Open rASIC1a Predicts a Binding Site Within the Pore. To reveal the diminazene and amiloride binding site at the atomic level, we docked both molecules to a homology model of rASIC1a in the open conformation based on the open conformation of chicken ASIC1 (Protein Data Bank accession number 4NTW) (Baconguis et al., 2014). As the voltage dependence of block and the competition between amiloride and diminazene strongly indicated a binding site within the membrane electric field and, thus, probably within the ion pore, we restricted the docking space to this area.

Molecular docking indeed located the diminazene and amiloride binding site within the outer half of the ion pore of rASIC1a (Fig. 6). We considered 15 and 16 binding poses within a $20-\mathrm{kcal} / \mathrm{mol}$ window from the lowest energy binding pose for amiloride and diminazene, respectively. Here, we examine the binding mode of the best poses according to the eModel score for both blockers (Supplemental Tables 1 and 2); binding modes of the other poses are reported in the Supplemental Material.

In its best binding pose, diminazene was surrounded (within $4 \AA$, chain indices reported in brackets) by residues $\mathrm{G} 431(\mathrm{C})$, D432(C), G434(B), G435(A-C), M437(B), G438(B and C), L439(A and C), G442(B), A443(B), and S444(B). Hydrogen bonds were formed between diminazene and the backbone of G434(B), G435(A), G438(C), A443(B), and with the side chain of L439(A and C). An H- $\pi$ interaction was found between G435(B) and the aromatic ring of diminazene. Further hydrophobic contacts were observed between diminazene and G431(C), G435(A and C), M437(B), G438(B), L439(C), and S444(B) (Fig. 6A-C). A protein ligand interaction fingerprint (PLIF) summarizing the interaction between blockers and ASIC1a using a fingerprint scheme was generated in the MOE (Molecular Operating Environment; Chemical Computing Group, Montreal, Quebec, Canada) (Vilar et al., 2008; Da and Kireev, 2014). The PLIF of the best diminazene binding pose showed that the residues mostly involved in binding are D432(C), G434(B), G435(A), G438(C), and L439(A and C), (Supplemental Fig. 2A). A PLIF involving all binding poses of diminazene was also generated (Supplemental Figs. 3 and 4) which showed a conservation of these interactions across most of the binding poses plus interactions with G442 and A443 for 8 out of 16 and 5 out of 16 binding poses, respectively. Interactions with E63 and Y67 were also found for two of the binding poses.

The best binding pose of amiloride was surrounded by residues D432(B), G434(A), G435(A-C), Q436(B and C), Q436(C), G438(A-C), and L439(B). Therefore, amiloride occupies the same binding region as diminazene, but being smaller, its binding region does not extend to the highly conserved GAS motif in the middle of TMD2 formed by G442, $\mathrm{A} 443$, and S444. Hydrogen bonds were formed between amiloride and the backbone of D432(B), G435(C), and G438(B), and with the side chain of Q436(C) and L439(B). Hydrophobic contacts were observed with residues $\mathrm{G} 435$ (B and $\mathrm{C}$ ), Q436(B), G438(B and C), and L439(B) (Fig. 6, D-F). For the best binding pose, a PLIF was generated, which showed that the residues mostly involved in binding of amiloride are D432(B), G435(B), Q436(B), G438(B), and L439(B) (Supplemental Fig. 2B). A PLIF involving all of the binding poses of amiloride was also generated (Supplemental Figs. 3 and 5), showing that the main interactions, with the exception of Q436(B), are mostly preserved in the other binding poses with an additional interaction of G431 for 3 out of 15 binding poses. 

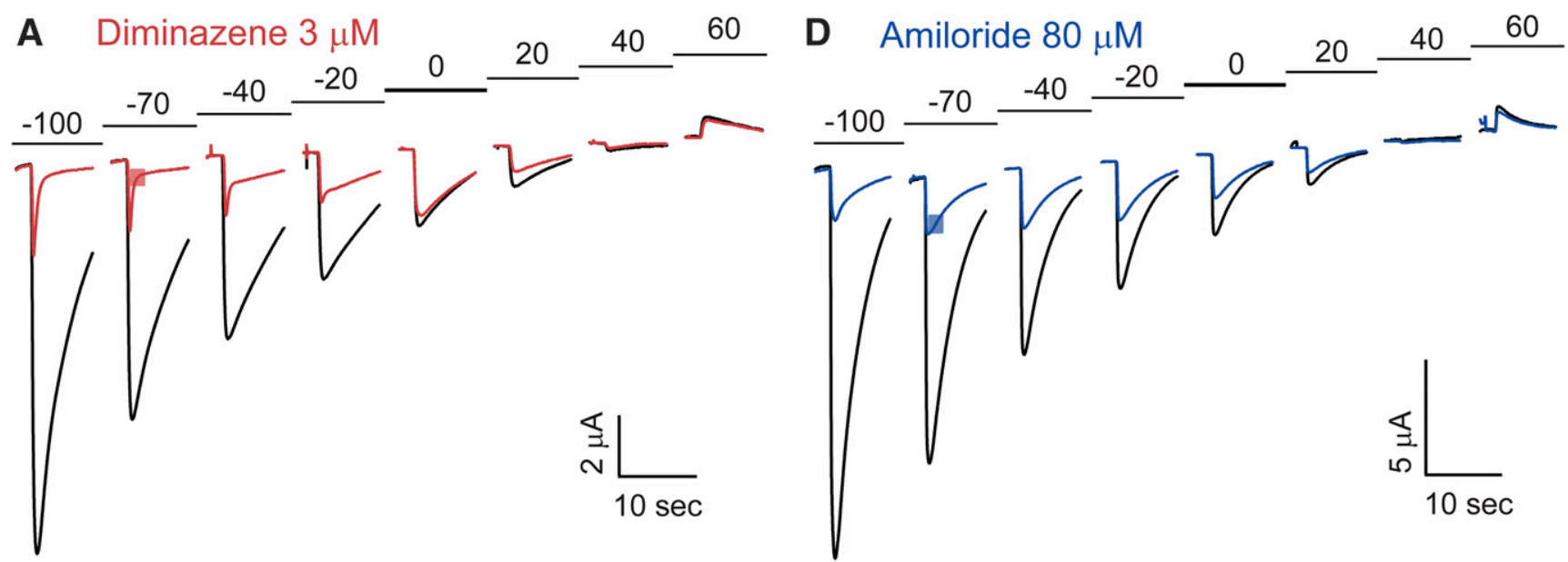

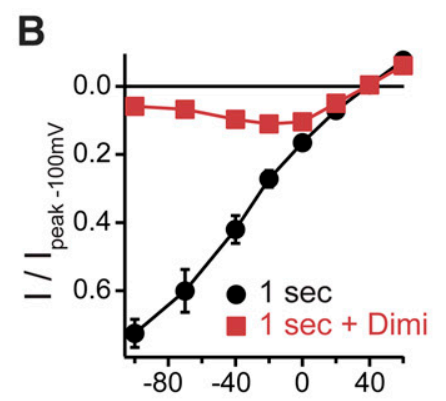

membrane potential $(\mathrm{mV})$

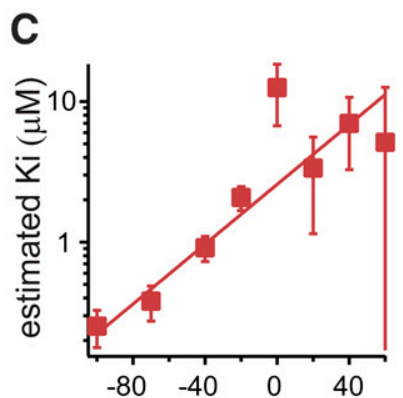

membrane potential $(\mathrm{mV})$

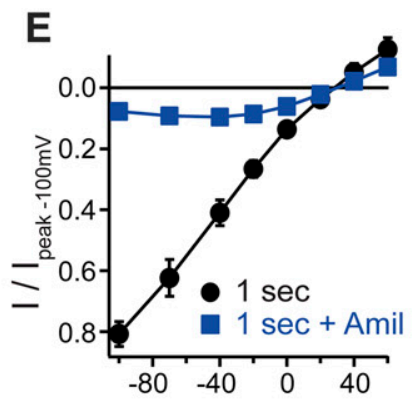

membrane potential $(\mathrm{mV})$

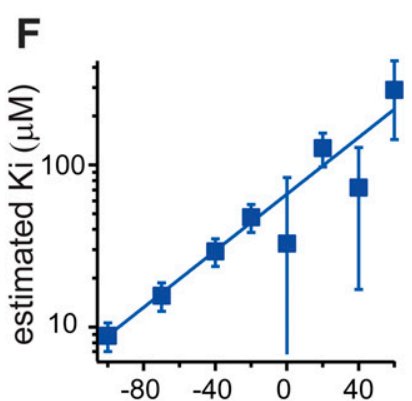

membrane potential $(\mathrm{mV})$

Fig. 5. Inhibition of rASIC1a by diminazene or amiloride is voltage-dependent. (A) Representative currents elicited by pH 6.5 in an rASIC1a-expressing oocyte in the absence (black) or presence (red) of $3 \mu \mathrm{M}$ diminazene at the indicated holding potentials (in millivolts). (B) I-V plot for rASIC1a in the absence (black) or presence (red) of diminazene. Currents 1 second postpeak [illustrated by the corresponding symbol in (A)] were normalized to the peak current at $-100 \mathrm{mV}$. (C) Estimated apparent inhibitory constants $\left(K_{\mathrm{i}}\right)$ of diminazene for currents 1 second postpeak are plotted against the holding potential (filled squares). Data were fit by the Woodhull model for channel block. To yield meaningful results despite variable error sizes, error weighted fits with the S.E.M. as input were used. Error bars represent S.E.M.; $n=8$. Representative current trace (D), I-V plot (E), and apparent $K_{\mathrm{i}}$ vs. voltage plots (F) for rASIC1a in the presence (blue) and absence (black) of $80 \mu \mathrm{M}$ amiloride. Error bars represent S.E.M.; $n=11$.

Therefore, molecular docking suggests overlapping binding sites of diminazene and amiloride within the outer half of the ASIC1a pore. Hence, this model can explain the competition of both molecules and the dependence of their inhibition on the membrane potential.

rASIC1a Pore Mutants G435C and L439C Strongly Decrease Diminazene and Amiloride Block. To test if molecular docking correctly predicted the binding site of diminazene and amiloride, we generated point mutations of residues adjacent to the predicted site in rASIC1a (G431C, G435C, Q436C, G438C, G438A, L439C, F440C, I441C, and $\mathrm{S} 444 \mathrm{~A}$ ) and tested the sensitivity of mutant channels to diminazene and amiloride. Similar mutations have previously already been used successfully to determine the shape of the ASIC1 ion pore (Li et al., 2011). ASIC1a wild type (wt) and mutants were activated by application of $\mathrm{pH} 6.5$ with and without pre- and coapplication of $3 \mu \mathrm{M}$ diminazene or $80 \mu \mathrm{M}$ amiloride, respectively (Fig. 7A). Diminazene and amiloride reduced current amplitudes of rASIC1a wt to $11.5 \% \pm 1.3 \%$ and $20.5 \% \pm 1.8 \%$, respectively, relative to control (1 second postpeak; Fig. 7B; $n=15$ ). In contrast, inhibition by diminazene was significantly reduced for the mutants G435C, Q436C, L439C, and I441C; mutants G435C and L439C were practically insensitive to diminazene (Fig. 7; G435C: $94.4 \% \pm 5.7 \%$ and L439C: $82.8 \% \pm 6.4 \%$ of control; $P<0.001 ; n=7-10)$. Both mutants were also nearly unaffected by amiloride (Fig. 7; G435C: $82.0 \% \pm 5.2 \%$ and L439C: $89.1 \% \pm 9.4 \%$ of control; $P<0.001 ; n=6-10)$. In addition, amiloride inhibition was also significantly reduced for Q436C, but not for I441C (Fig. 7B). Interestingly, mutant G431C was slightly more strongly inhibited by both inhibitors (Fig. 7B; diminazene: $5.9 \% \pm 1.4 \%$; G431C vs. wt: $P=0.06$; amiloride: $8.2 \% \pm 1.6 \%$; G431C vs. wt: $P=0.001 ; n=8)$. Similarly, mutant F440C was more strongly inhibited by amiloride $(10.6 \% \pm 3.1 \% ; P=0.051 ; n=7)$, but not diminazene $(23.3 \% \pm 8.3 \% ; P=0.34 ; n=7)$. Mutations G438C, G438A, and S444A led to a loss of function, as we never observed $\mathrm{pH}$-activated currents in oocytes injected with the respective cRNA.

As G435, G438, and L439 were predicted to form hydrogen bonds with both amiloride and diminazene (Fig. 6), and mutations of the residues G435 and L439 dramatically reduced the inhibition by both blockers, these results strongly support our docking results. Taken together, our results strongly support a diminazene and amiloride binding site within the outer half of the ASIC1a pore.

\section{Discussion}

There were two main findings in this study. First, a detailed analysis of the inhibition of ASIC1a by diminazene revealed 
A

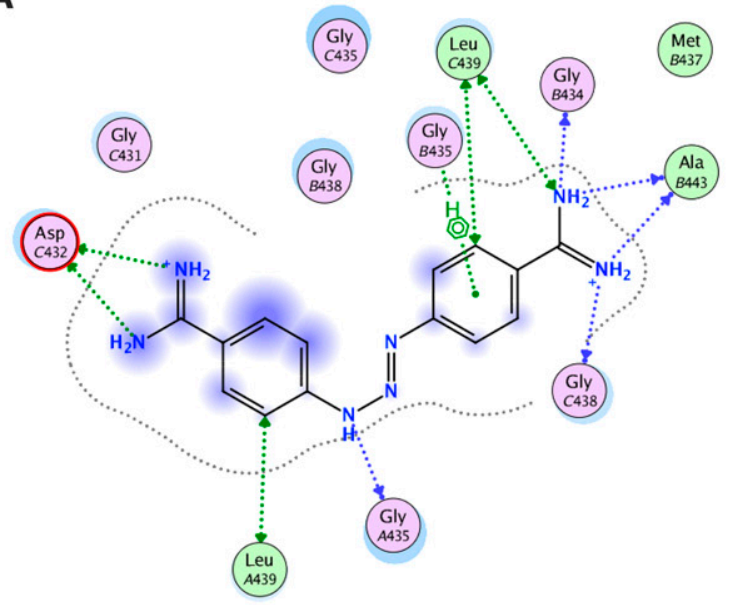

B

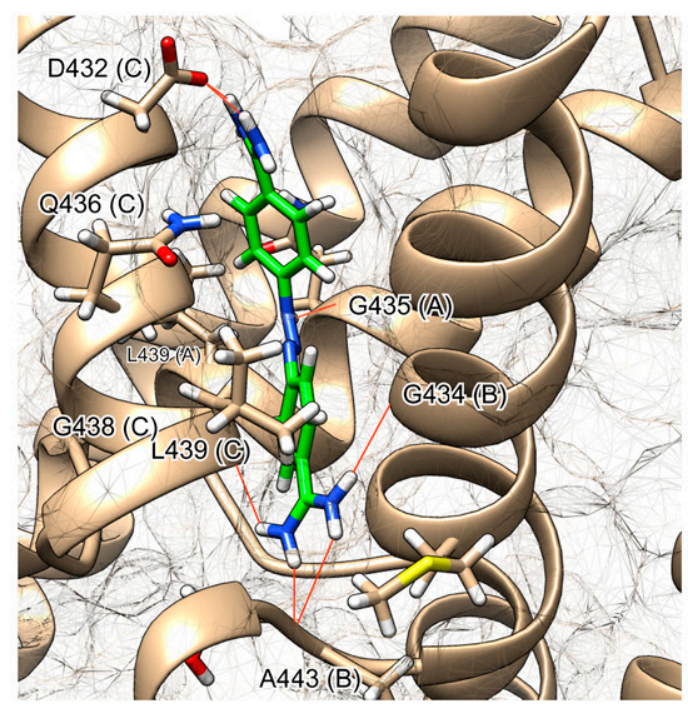

C

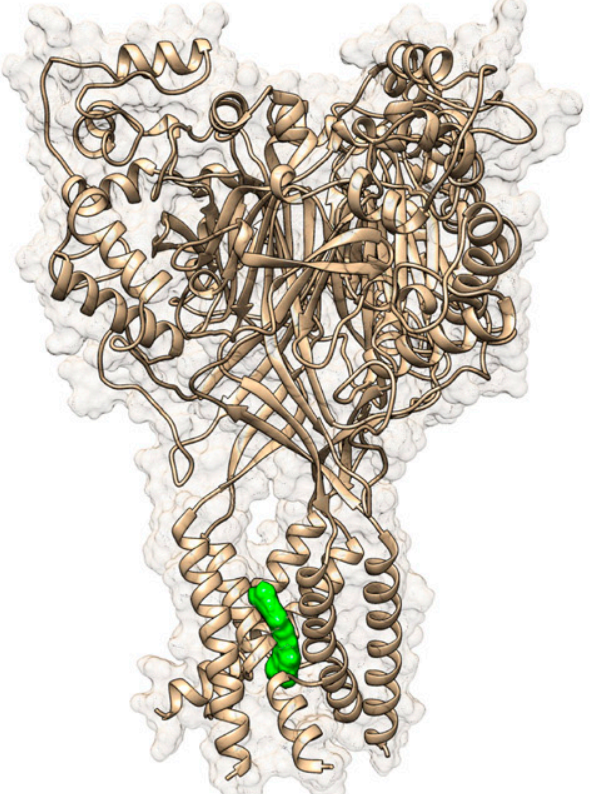

D
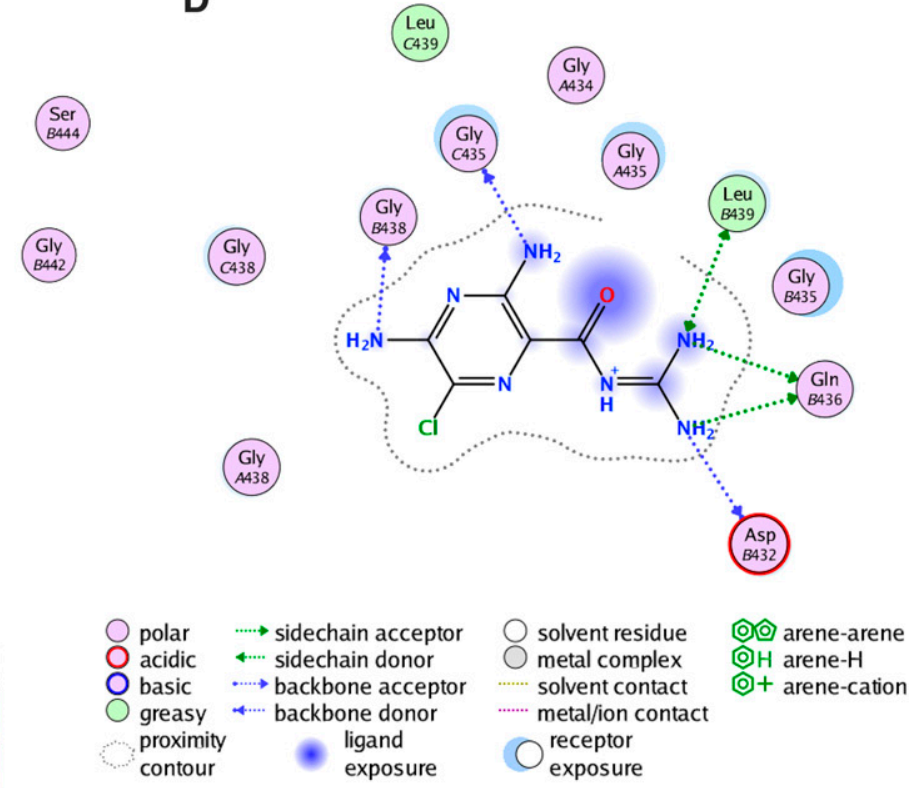

E

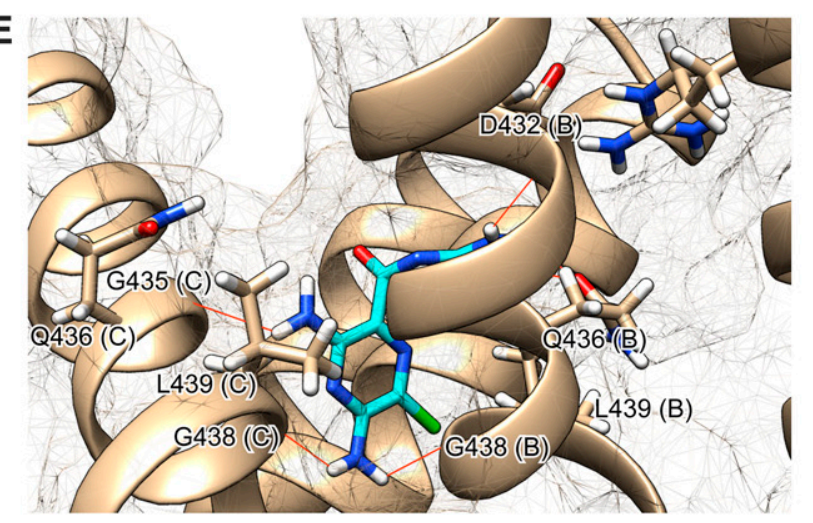

F

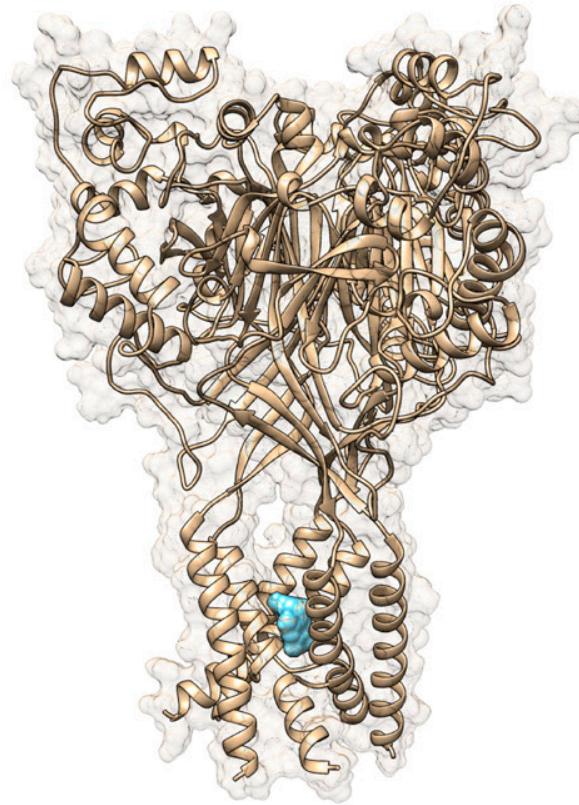

Fig. 6. Molecular docking predicts a diminazene binding site within the pore. (A and D) Two-dimensional ligand interaction diagram of the best binding pose for diminazene (A) and amiloride (D). Key amino acids within $4 \AA$ of docked compounds and their binding interactions were identified. (B and $\mathrm{E}$ ) Local tridimensional view of ligands within the ASIC1a binding pocket. The ligands are in green (B) and cyan (E) licorice representation for diminazene and amiloride, respectively. (C and F) Full tridimensional view of ASIC1a with bound diminazene (C) or amiloride (F) in green and cyan solid surface representation, respectively. The protein is represented as an illustration with a wireframe surface. 

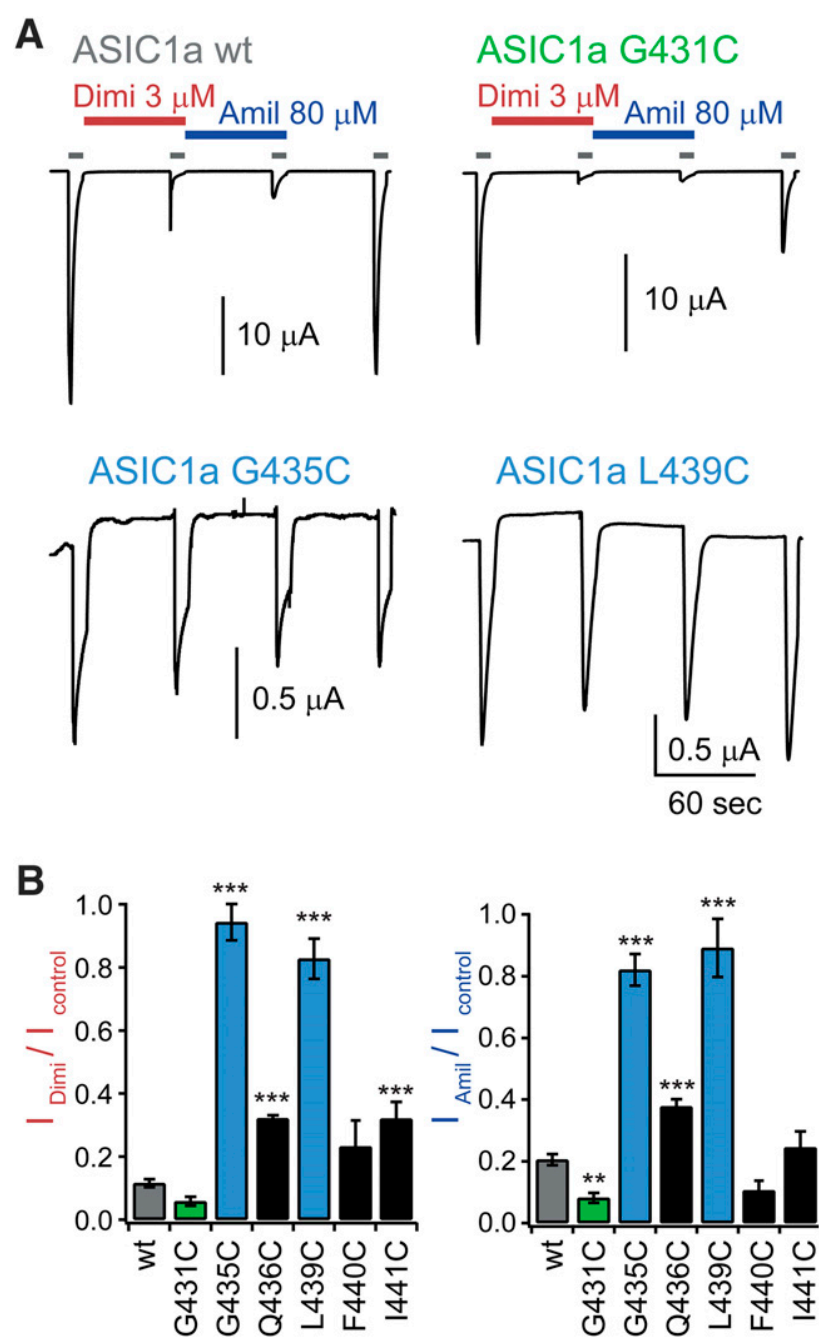

Fig. 7. Functional effect of mutations of residues surrounding the predicted diminazene and amiloride binding site. (A) Representative current traces of ASIC1a wild type, ASIC1a G431C, ASIC1a G435C, and ASIC1a L439C repeatedly activated by $\mathrm{pH} 6.5$ in the absence or presence of $3 \mu \mathrm{M}$ diminazene (Dimi, red bar) or $80 \mu \mathrm{M}$ amiloride (Amil, blue bar). (B) Current amplitudes 1 second postpeak in the presence of $3 \mu \mathrm{M}$ diminazene (left) or $80 \mu \mathrm{M}$ amiloride (right) normalized to the first current amplitude in the absence of an inhibitor. $* * P<0.01$; $* * * P<0.001$ (paired Student's $t$ test). Error bars represent S.E.M.; $n=6-15$.

that diminazene acts as a slow blocker of the open state of ASIC1a. Second, functional analysis combined with molecular docking and site-directed mutagenesis consistently showed that diminazene blocks ASIC1a by binding within the ion pore above the "GAS belt," which constricts the open pore (Baconguis et al., 2014) but may not be the selectivity filter of ASICs (Lynagh et al., 2017). Diminazene binds to a site that largely overlaps with the binding site of amiloride, but with higher affinity than amiloride. In the original discovery of diminazene as a potent inhibitor of ASICs, it was proposed that it binds within a small groove in the ECD, formed by part of the beta-ball domain and the palm domain (Chen et al., 2010b). Binding to other domains could not be excluded, however, and binding to the groove in the ECD was not confirmed experimentally (Chen et al., 2010b). Our study resolves this uncertainty and clearly reveals the diminazene binding site, which is responsible for high-affinity inhibition of ASIC1a. However, we cannot exclude that diminazene also binds with lower affinity to other sites on the channel, such as in the proposed groove in the ECD.

Based on voltage dependence of inhibition and competition with amiloride, a pore-block mechanism of diminazene was previously already suggested for the bile acid-sensitive ion channel (BASIC; also named ASIC5) (Wiemuth and Gründer, 2011), which is closely related to ASICs. The findings of the present study are thus in good agreement with the previous findings on BASIC and suggest a conserved structure of the outer pore of ASICs and BASIC. In addition to the previous findings on BASIC, the present study describes in molecular detail the binding site within the outer ion pore and identifies residues G435 and L439 as particularly important contact points of diminazene.

Peptide-gated ion channels from Hydra, the $\mathrm{HyNaCs}$, which are also closely related to ASIC/BASIC (Golubovic et al., 2007), are also inhibited by diminazene with an apparent affinity that varies for different $\mathrm{HyNaCs}$ between 0.05 and $31 \mu \mathrm{M}$ and is $>100$-fold higher than the apparent affinity of amiloride at these channels (Assmann et al., 2014). Although the mechanism of inhibition of diminazene has not been investigated, it is highly likely that it is also a pore blocker of $\mathrm{HyNaCs}$.

Although the $\mathrm{ENaC}$ belongs to the same gene family as ASICs/BASIC and HyNaCs, it is not closely related to these channels (Assmann et al., 2014), and its pore-blocker pharmacology has some remarkable differences: it is inhibited by amiloride with high affinity (Canessa et al., 1994), comparable to that of diminazene at ASICs, but it is not inhibited by diminazene (Chen et al., 2010b), highlighting substantial differences in the structure of the outer pore of ASICs/BASIC/ $\mathrm{HyNaCs}$ and ENaC.

In addition to reducing the peak current amplitude of ASICs, diminazene has the interesting property of leading to an apparent acceleration of desensitization of the remaining current (Chen et al., 2010b). We could confirm this rapid decline of the current, which was initially described for ASICs from mouse hippocampal neurons (Chen et al., 2010b), for rat ASIC1a. It was proposed that diminazene facilitated desensitization, and that it inhibits ASIC currents via a distinct mechanism compared with amiloride (Chen et al., 2010b). Our study, however, revealed that diminazene and amiloride both inhibit ASICs via a common pore-block mechanism. As the outer pore constricts during desensitization (Gonzales et al., 2009; Gründer and Augustinowski, 2012), an acceleration of desensitization by diminazene is incompatible with its binding site within the ion pore. We could indeed show that diminazene stabilizes the open state of ASIC1a and slows, rather than accelerates, desensitization, as expected for an open-channel blocker. Collectively, our results thus suggest that diminazene slowly binds to its binding site in the ion pore, leading to an incomplete block at the onset of activation and to the rapid decline of the peak current. Such a scenario implies that the analysis of peak current amplitudes underestimates the potency of diminazene at ASICs, and we could indeed show that the apparent inhibitory constant $\left(K_{\mathrm{i}}\right)$ determined 1 second after peak is $0.3 \mu \mathrm{M}$, approximately 3 -fold higher than the potency determined at peak.

Using diminazene to inhibit ASICs in vivo, it is hard to predict whether the incomplete inhibition of the peak current by diminazene would be more relevant than the complete inhibition after few seconds. Although ASICs are supposed to open only briefly during synaptic stimulation (MacLean and 
Jayaraman, 2016), for example (not allowing for a complete block by diminazene), during high-frequency stimulation of ASICs, a cumulative slow block by diminazene might still reduce ASIC currents more strongly than predicted by the reduction of peak current amplitudes. This is also the case in situations in which the $\mathrm{pH}$ drops for longer time periods, such as during ischemia or peripheral inflammation. Irrespective of these uncertainties, a blocker with the same affinity as diminazene but a faster on-rate would be desirable for clinical use.

Nafamostat mesilate is chemically related to diminazene (Chen et al., 2010a) and blocks ASIC1a with an apparent $K_{\mathrm{i}}$ of $15 \mu \mathrm{M}$; its affinity to $\mathrm{ENaC}$, however, is low $\left(K_{\mathrm{i}}>1 \mathrm{mM}\right)$ (Ugawa et al., 2007). These properties suggest that it may share the binding site with diminazene (Rash, 2017). For BASIC, it has indeed been shown that block by nafamostat is voltagedependent (Wiemuth and Gründer, 2011). We therefore speculate that nafamostat is also an open-channel blocker of ASICs. As diminazene has a significantly higher potency at ASICs than nafamostat, our results indicate that diminazene is an interesting lead compound for unspecific blockers of ASICs.

\section{Acknowledgments}

The authors thank A. Oslender-Bujotzek for expert technical assistance and D. Wiemuth and M. Pusch for helpful discussions.

\section{Authorship Contributions}

Participated in research design: Schmidt, Gründer.

Conducted experiments: Schmidt, Joussen.

Performed data analysis: Schmidt, Rossetti.

Wrote or contributed to the writing of the manuscript: Schmidt, Gründer.

\section{References}

Askwith CC, Wemmie JA, Price MP, Rokhlina T, and Welsh MJ (2004) Acid-sensing ion channel 2 (ASIC2) modulates ASIC1 $\mathrm{H}+$-activated currents in hippocampal neurons. J Biol Chem 279:18296-18305.

Assmann M, Kuhn A, Dürrnagel S, Holstein TW, and Gründer S (2014) The comprehensive analysis of $\mathrm{DEG} / \mathrm{ENaC}$ subunits in Hydra reveals a large variety of peptide-gated channels, potentially involved in neuromuscular transmission. BMC Biol 12:84

Baconguis I, Bohlen CJ, Goehring A, Julius D, and Gouaux E (2014) X-ray structure of acid-sensing ion channel 1-snake toxin complex reveals open state of a $\mathrm{Na}(+)$-selective channel. Cell 156:717-729.

Baron A and Lingueglia E (2015) Pharmacology of acid-sensing ion channels - Physiological and therapeutical perspectives. Neuropharmacology 94:19-35.

Baron A, Waldmann R, and Lazdunski M (2002) ASIC-like, proton-activated currents in rat hippocampal neurons. J Physiol 539:485-494.

Bartoi T, Augustinowski K, Polleichtner G, Gründer S, and Ulbrich MH (2014) Acidsensing ion channel (ASIC) 1a/2a heteromers have a flexible 2:1/1:2 stoichiometry. Proc Natl Acad Sci USA 111:8281-8286.

Bässler EL, Ngo-Anh TJ, Geisler HS, Ruppersberg JP, and Gründer S (2001) Molecular and functional characterization of acid-sensing ion channel (ASIC) 1b. J Biol Chem 276:33782-33787.

Canessa CM, Schild L, Buell G, Thorens B, Gautschi I, Horisberger JD, and Rossier $\mathrm{BC}$ (1994) Amiloride-sensitive epithelial $\mathrm{Na}+$ channel is made of three homologous subunits. Nature 367:463-467.

Chassagnon IR, McCarthy CA, Chin YK, Pineda SS, Keramidas A, Mobli M, Pham V, De Silva TM, Lynch JW, Widdop RE, et al. (2017) Potent neuroprotection after stroke afforded by a double-knot spider-venom peptide that inhibits acid-sensing ion channel 1a. Proc Natl Acad Sci USA 114:3750-3755.

Chen X, Orser BA, and MacDonald JF (2010a) Design and screening of ASIC inhibitors based on aromatic diamidines for combating neurological disorders. Eur $J$ Pharmacol 648:15-23.

Chen X, Qiu L, Li M, Dürrnagel S, Orser BA, Xiong ZG, and MacDonald JF (2010b) Diarylamidines: high potency inhibitors of acid-sensing ion channels. Neuropharmacology 58:1045-1053.

Da C and Kireev D (2014) Structural protein-ligand interaction fingerprints (SPLIF) for structure-based virtual screening: method and benchmark study. J Chem Inf Model 54:2555-2561.

Deval E and Lingueglia E (2015) Acid-sensing ion channels and nociception in the peripheral and central nervous systems. Neuropharmacology 94:49-57.

Deval E, Noël J, Lay N, Alloui A, Diochot S, Friend V, Jodar M, Lazdunski M, and Lingueglia E (2008) ASIC3, a sensor of acidic and primary inflammatory pain. EMBO J 27:3047-3055.

Diochot S, Alloui A, Rodrigues P, Dauvois M, Friend V, Aissouni Y, Eschalier A, Lingueglia E, and Baron A (2016) Analgesic effects of mambalgin peptide inhibitors of acid-sensing ion channels in inflammatory and neuropathic pain. Pain 157:552-559.

Diochot S, Baron A, Salinas M, Douguet D, Scarzello S, Dabert-Gay AS, Debayle D Friend V, Alloui A, Lazdunski M, et al. (2012) Black mamba venom peptides target acid-sensing ion channels to abolish pain. Nature 490:552-555.

Dorofeeva NA, Barygin OI, Staruschenko A, Bolshakov KV, and Magazanik LG (2008) Mechanisms of non-steroid anti-inflammatory drugs action on ASICs expressed in hippocampal interneurons. J Neurochem 106:429-441.

Du J, Reznikov LR, Price MP, Zha XM, Lu Y, Moninger TO, Wemmie JA, and Welsh MJ (2014) Protons are a neurotransmitter that regulates synaptic plasticity in the lateral amygdala. Proc Natl Acad Sci USA 111:8961-8966.

Dubé GR, Lehto SG, Breese NM, Baker SJ, Wang X, Matulenko MA, Honoré P, Stewart AO, Moreland RB, and Brioni JD (2005) Electrophysiological and in vivo characterization of A-317567, a novel blocker of acid sensing ion channels. Pain 117:88-96.

Friese MA, Craner MJ, Etzensperger R, Vergo S, Wemmie JA, Welsh MJ, Vincent A, and Fugger L (2007) Acid-sensing ion channel-1 contributes to axonal degeneration in autoimmune inflammation of the central nervous system. Nat Med 13 1483-1489.

Friesner RA, Banks JL, Murphy RB, Halgren TA, Klicic JJ, Mainz DT, Repasky MP, Knoll EH, Shelley M, Perry JK, et al. (2004) Glide: a new approach for rapid, accurate docking and scoring. 1. Method and assessment of docking accuracy. $J$ Med Chem 47:1739-1749.

Gallicchio E, Zhang LY, and Levy RM (2002) The SGB/NP hydration free energy model based on the surface generalized born solvent reaction field and novel nonpolar hydration free energy estimators. J Comput Chem 23:517-529.

Ghosh A, Rapp CS, and Friesner RA (1998) Generalized born model based on a surface integral formulation. J Phys Chem B 102:10983-10990.

Golubovic A, Kuhn A, Williamson M, Kalbacher H, Holstein TW, Grimmelikhuijzen CJ, and Gründer S (2007) A peptide-gated ion channel from the freshwater polyp Hydra. J Biol Chem 282:35098-35103.

Gonzales EB, Kawate T, and Gouaux E (2009) Pore architecture and ion sites in acidsensing ion channels and P2X receptors. Nature 460:599-604.

González-Inchauspe C, Urbano FJ, Di Guilmi MN, and Uchitel OD (2017) Acidsensing ion channels activated by evoked released protons modulate synaptic transmission at the mouse calyx of held synapse. J Neurosci 37:2589-2599.

Greenwood JR, Calkins D, Sullivan AP, and Shelley JC (2010) Towards the comprehensive, rapid, and accurate prediction of the favorable tautomeric states of drug-like molecules in aqueous solution. J Comput Aided Mol Des 24:591-604.

Gründer S and Augustinowski K (2012) Toxin binding reveals two open state structures for one acid-sensing ion channel. Channels (Austin) 6:409-413.

Gründer S, Geissler HS, Bässler EL, and Ruppersberg JP (2000) A new member of acid-sensing ion channels from pituitary gland. Neuroreport 11:1607-1611.

Gründer S and Pusch M (2015) Biophysical properties of acid-sensing ion channels (ASICs). Neuropharmacology 94:9-18.

Halgren TA, Murphy RB, Friesner RA, Beard HS, Frye LL, Pollard WT, and Banks JL (2004) Glide: a new approach for rapid, accurate docking and scoring. 2. Enrichment factors in database screening. $J$ Med Chem 47:1750-1759.

Harder E, Damm W, Maple J, Wu C, Reboul M, Xiang JY, Wang L, Lupyan D, Dahlgren MK, Knight JL, et al. (2016) OPLS3: a force field providing broad coverage of drug-like small molecules and proteins. $J$ Chem Theory Comput 12 281-296.

Huang Y, Jiang N, Li J, Ji YH, Xiong ZG, and Zha XM (2015) Two aspects of ASIC function: synaptic plasticity and neuronal injury. Neuropharmacology 94:42-48.

Jacobson MP, Friesner RA, Xiang Z, and Honig B (2002) On the role of the crystal environment in determining protein side-chain conformations. $J$ Mol Biol 320: $597-608$

Jasti J, Furukawa H, Gonzales EB, and Gouaux E (2007) Structure of acid-sensing ion channel 1 at $1.9 \mathrm{~A}$ resolution and low $\mathrm{pH}$. Nature 449:316-323.

Joeres N, Augustinowski K, Neuhof A, Assmann M, and Gründer S (2016) Functional and pharmacological characterization of two different ASIC1a/2a heteromers reveals their sensitivity to the spider toxin PcTx1. Sci Rep 6:27647.

Jorgensen WL, Maxwell DS, and Tirado-Rives J (1996) Development and testing of the OPLS all-atom force field on conformational energetics and properties of organic liquids. J Am Chem Soc 118:11225-11236.

Kellenberger S and Schild L (2015) International Union of Basic and Clinical Pharmacology. XCI. structure, function, and pharmacology of acid-sensing ion channels and the epithelial Na+ channel. Pharmacol Rev 67:1-35.

Kleyman TR and Cragoe EJ, Jr (1988) Amiloride and its analogs as tools in the study of ion transport. J Membr Biol 105:1-21.

Kreple CJ, Lu Y, Taugher RJ, Schwager-Gutman AL, Du J, Stump M, Wang Y, Ghobbeh A, Fan R, Cosme CV, et al. (2014) Acid-sensing ion channels contribute to synaptic transmission and inhibit cocaine-evoked plasticity. Nat Neurosci 17: 1083-1091.

Li T, Yang Y, and Canessa CM (2011) Outlines of the pore in open and closed conformations describe the gating mechanism of ASIC1. Nat Commun 2:399.

Lovell SC, Davis IW, Arendall WB, III, de Bakker PIW, Word JM, Prisant MG, Richardson JS, and Richardson DC (2003) Structure validation by Calpha geometry: $\varphi, \psi$ and Cbeta deviation. Proteins 50:437-450.

Lynagh T, Flood E, Boiteux C, Wulf M, Komnatnyy VV, Colding JM, Allen TW, and Pless SA (2017) A selectivity filter at the intracellular end of the acid-sensing ion channel pore. eLife $\mathbf{6}$

MacLean DM and Jayaraman V (2016) Acid-sensing ion channels are tuned to follow high-frequency stimuli. J Physiol 594:2629-2645.

Madeja M, Musshoff U, and Speckmann EJ (1995) Improvement and testing of a concentration-clamp system for oocytes of Xenopus laevis. J Neurosci Methods 63: 211-213

Mazzuca M, Heurteaux C, Alloui A, Diochot S, Baron A, Voilley N, Blondeau N, Escoubas P, Gélot A, Cupo A, et al. (2007) A tarantula peptide against pain via ASIC1a channels and opioid mechanisms. Nat Neurosci 10:943-945. 
McCarthy CA, Rash LD, Chassagnon IR, King GF, and Widdop RE (2015) PcTx1 affords neuroprotection in a conscious model of stroke in hypertensive rats via selective inhibition of ASIC1a. Neuropharmacology 99:650-657.

Molliver DC, Immke DC, Fierro L, Paré M, Rice FL, and McCleskey EW (2005) ASIC3, an acid-sensing ion channel, is expressed in metaboreceptive sensory neurons. Mol Pain 1:35.

O’Boyle NM, Banck M, James CA, Morley C, Vandermeersch T, and Hutchison GR (2011) Open babel: an open chemical toolbox. J Cheminform 3:33.

Palmer LG (1984) Voltage-dependent block by amiloride and other monovalent cations of apical Na channels in the toad urinary bladder. J Membr Biol 80:153-165.

Rash LD (2017) Acid-sensing ion channel pharmacology, past, present, and future ... Adv Pharmacol 79:35-66.

Sali A and Blundell TL (1993) Comparative protein modelling by satisfaction of spatial restraints. J Mol Biol 234:779-815.

Sastry GM, Adzhigirey M, Day T, Annabhimoju R, and Sherman W (2013) Protein and ligand preparation: parameters, protocols, and influence on virtual screening enrichments. J Comput Aided Mol Des 27:221-234.

Saugstad JA, Roberts JA, Dong J, Zeitouni S, and Evans RJ (2004) Analysis of the membrane topology of the acid-sensing ion channel 2a. J Biol Chem 279 : $55514-55519$.

Schild L, Schneeberger E, Gautschi I, and Firsov D (1997) Identification of amino acid residues in the alpha, beta, and gamma subunits of the epithelial sodium channel (ENaC) involved in amiloride block and ion permeation. J Gen Physiol 109 $15-26$

Shelley JC, Cholleti A, Frye LL, Greenwood JR, Timlin MR, and Uchimaya M (2007) Epik: a software program for $\mathrm{pK}$ ( a ) prediction and protonation state generation for drug-like molecules. J Comput Aided Mol Des 21:681-691.

Shen MY and Sali A (2006) Statistical potential for assessment and prediction of protein structures. Protein Sci 15:2507-2524.

Sherman W, Day T, Jacobson MP, Friesner RA, and Farid R (2006) Novel procedure for modeling ligand/receptor induced fit effects. J Med Chem 49:534-553.

Sherwood TW, Lee KG, Gormley MG, and Askwith CC (2011) Heteromeric acidsensing ion channels (ASICs) composed of ASIC2b and ASIC1a display nove channel properties and contribute to acidosis-induced neuronal death. $J$ Neurosci 31:9723-9734.
Ugawa S, Ishida Y, Ueda T, Inoue K, Nagao M, and Shimada S (2007) Nafamostat mesilate reversibly blocks acid-sensing ion channel currents. Biochem Biophys Res Commun 363:203-208.

Vilar S, Cozza G, and Moro S (2008) Medicinal chemistry and the molecular operating environment (MOE): application of QSAR and molecular docking to drug discovery. Curr Top Med Chem 8:1555-1572.

Vukicevic M and Kellenberger S (2004) Modulatory effects of acid-sensing ion channels on action potential generation in hippocampal neurons. Am J Physiol Cell Physiol 287:C682-C690.

Waldmann R, Champigny G, Bassilana F, Heurteaux C, and Lazdunski M (1997) A proton-gated cation channel involved in acid-sensing. Nature 386:173-177.

Wemmie JA, Taugher RJ, and Kreple CJ (2013) Acid-sensing ion channels in pain and disease. Nat Rev Neurosci 14:461-471.

Wiemuth D and Gründer S (2011) The pharmacological profile of brain liver intestine $\mathrm{Na}+$ channel: inhibition by diarylamidines and activation by fenamates. $M o l$ Pharmacol 80:911-919.

Woodhull AM (1973) Ionic blockage of sodium channels in nerve. J Gen Physiol 61 687-708.

Wu LJ, Duan B, Mei YD, Gao J, Chen JG, Zhuo M, Xu L, Wu M, and Xu TL (2004) Characterization of acid-sensing ion channels in dorsal horn neurons of rat spinal cord. J Biol Chem 279:43716-43724.

Xiong ZG, Zhu XM, Chu XP, Minami M, Hey J, Wei WL, MacDonald JF, Wemmie JA, Price MP, Welsh MJ, et al. (2004) Neuroprotection in ischemia: blocking calciumpermeable acid-sensing ion channels. Cell 118:687-698.

Yen YT, Tu PH, Chen CJ, Lin YW, Hsieh ST, and Chen CC (2009) Role of acidsensing ion channel 3 in sub-acute-phase inflammation. Mol Pain 5:1.

Address correspondence to: Axel Schmidt, Institute of Physiology, RWTH Aachen University, Pauwelsstrasse 30, D-52074 Aachen, Germany. E-mail: axel.schmidt1@rwth-aachen.de; or Stefan Gründer, Institute of Physiology, RWTH Aachen University, Pauwelsstrasse 30, D-52074 Aachen, Germany. E-mail: sgruender@ukaachen.de 